Article

\title{
Acetyltransferases GCN5 and PCAF are required for B lympho- cyte maturation in mice
}

\author{
Valentyn Oksenych ${ }^{1,2,3,4 *}$, Dan Su${ }^{1}$, Jeremy A. Daniel ${ }^{1 *}$ \\ 1 The NNF Center for Protein Research, Faculty of Health and Medical Sciences, University of Copenhagen, \\ Blegdamsvej 3B, 2200 Copenhagen, Denmark; sudan20072007@yahoo.com \\ 2 Department for Cancer Research and Molecular Medicine (IKOM), Norwegian University of Science and \\ Technology, Laboratory Center, Erling Skjalgssons gate 1, 7491 Trondheim, Norway; valentyn.okse- \\ nych@ntnu.no \\ 3 Current address : KG Jebsen Centre for B Cell Malignancies, Institute of Clinical Medicine, University of \\ Oslo, N-0316 Oslo, Norway; \\ 4 Current address : Institute of Clinical Medicine, University of Oslo, 0318 Oslo, Norway; \\ valentyn.oksenych@medisin.uio.no \\ * Correspondence: Valentyn Oksenych, valentyn.oksenych@medisin.uio.no
}

\begin{abstract}
B lymphocyte development has two DNA recombination processes: V(D)J recombination of the immunoglobulin (Igh) gene variable region and class switching of the Igh constant regions from IgM to IgG, IgA, or IgE. V(D)J recombination is required for successful maturation of B cells from pro-B to pre-B to immature-B and then to mature B cells in the bone marrow. CSR occurs outside of the bone marrow when mature B cells migrate to peripheral lymphoid organs, such as spleen and lymph nodes. Both V(D)J recombination and CSR depend on an open chromatin state that makes DNA accessible to specific enzymes, recombination activating gene (RAG), and activation-induced cytidine deaminase (AID). Acetyltransferases GCN5 and PCAF possess redundant functions acetylating histone $\mathrm{H} 3$ lysine 9 (H3K9). Here, we generated a mouse model that lacks both GCN5 and PCAF in B cells. We found that double-deficient mice possess low levels of mature B cells in the bone marrow and peripheral organs, an accumulation of pro-B cells in bone marrow, and reduced CSR levels. We conclude that both GCN5 and PCAF are required for B cell development in vivo.
\end{abstract}

Keywords: KAT2A; KAT2B; mice; acetyltransferase; B cell; lymphocyte; class switching

\section{Introduction}

Development of B lymphocytes starts in the bone marrow where progenitor (pro)-B cells using recombination-activating genes (RAG) generate DNA double-strand breaks (DSBs) and initiate the V(D)J recombination (1). In maturating B cells, the $\mathrm{V}(\mathrm{D}) \mathrm{J}$ recombination process is genetic recombination of variable $(V)$, diversity $(D)$, and joining $(J)$ gene segments arranging into a newly formed VDJ part of immunoglobulin gene (Ig) (2-6). Following the $\mathrm{V}(\mathrm{D}) \mathrm{J}$ recombination, $\mathrm{B}$ cells develop from pro-B cells expressing specific markers cluster of differentiation 19 (CD19), B220/CD45, and CD43 (CD19+B220+CD43+) to pre-B cells (CD19+B220+CD43-), immature B (CD19+B220+IgM+, low immunoglobulin $\mathrm{M}, \mathrm{IgM})$ and mature $\mathrm{B}(\mathrm{CD} 19+\mathrm{B} 220+\mathrm{IgM}+$, high $\mathrm{IgM})$ cells in bone marrow (2). Mature $\mathrm{B}$ lymphocytes leave the bone marrow and through the blood migrate to periphery populating spleen and lymph nodes.

Then, mature B cells initiate another DNA recombination process to change the constant regions of immunoglobulin genes referred to as class switch recombination (CSR). During the CSR in mice, IgM is replaced by IgG3, IgG1, IgG2a, IgG2b, IgE, or IgA (2). The CSR is initiated by non-productive transcription known as a germ-line transcription (GLT) which is needed to separate two DNA strands. Single-stranded DNA is then targeted by 
activation-induced cytidine deaminase (AID), a B lymphocyte-specific enzyme deaminating cytosine to uracil ( $\mathrm{C}$ to $\mathrm{U}$ ). Then uracil DNA N-glycosylase (UNG) removes the uracil from DNA leading to the single-strand break formation (SSB)(7). Two SSBs facing each other then form a DSB and allow recombination $(2-5,8)$. Both V(D)J and CSR can be regarded as processes following fundamentally similar strategies of genomic recombination $(9,10)$.

The DSBs formed during the $\mathrm{V}(\mathrm{D}) \mathrm{J}$ recombination and CSR are recognized, processed, and repaired by the non-homologous end-joining pathway (NHEJ) and initiate more complex signaling and chromatin modification pathway known as DNA damage response (DDR) (2-5). The NHEJ is initiated when Ku70 and Ku80 heterodimer (Ku) recognizes and binds the DSBs. Ku serves as a platform for downstream factors including DNAdependent protein kinase catalytic subunit (DNA-PKcs), X-ray repair cross-complementing protein 4 (XRCC4), XRCC4-like factor (XLF), Paralogue of XRCC4 and XLF (PAXX), a modulator of retrovirus infection (MRI), and DNA ligase 4 (LIG4) $(3-5,11)$. There are additional factors that are sometimes optional for NHEJ, including Artemis with nuclease activity required for processing hairpin-sealed DNA ends and overhangs $(2,5,12)$.

One type of DDR pathway acts downstream of ataxia telangiectasia mutated (ATM) protein kinase, which is activated by DSBs and then phosphorylates multiple substrates, including NHEJ and DDR factors. ATM phosphorylates histone H2AX, which in turn recruits mediator of DNA damage checkpoint 1 (MDC1) and facilitates the accumulation of really interesting new gene (RING) finger motif (RNF) 8 and RNF168 ubiquitin-ligases, and then the p53-binding protein (53BP1). Phosphorylation of H2AX is related to the acetylation of histones, including histone $\mathrm{H} 3 \mathrm{~K} 9$. In particular, histone acetylation relies on the ATM-dependent H2AX phosphorylation and SWI/SNF chromatin remodeling factors (13). Acetylation of histone H3K9 is mediated by GCN5 and PCAF $(13,14)$.

There is a complex genetic interaction associated with the functional redundancy between the NHEJ factors $(3,4)$, including the following pairs: DNA-PKcs/XLF $(5,15-17)$, PAXX/XLF (18-24), and MRI/XLF $(25,26)$. Moreover, there is a genetic interaction between NHEJ and DDR pathway factors, for example, ATM/XLF and H2AX/XLF (27), MDC1/XLF (28), RNF8/XLF and RNF168/XLF (29), 53BP1/XLF (30, 31), and more (3, 4). Moreover, acetyltransferases GCN5 and PCAF are redundant in promoting histone H3 lysine K9 acetylation (32).

General control non-depressible 5 (GCN5) acetyltransferase is also known as lysine acetyltransferase (KAT) 2A. Germline inactivation of GCN5 in mice resulted in early embryonic lethality due to the role of the protein in neurogenesis (32). GCN5 is functionally redundant with another acetyltransferase, KAT2B, also referred to as p300/CBP-associated factor (PCAF). While inactivation of Pcaf gene in mice has no detectable phenotype, double knockout of Gcn5/Pcaf genes resulted in even earlier embryonic lethality than in Gcn5/mice (32). Because histone H3K9 acetylation works downstream of ATM and H2AX in DDR (13), one could propose that GCN5, PCAF, or both enzymes are required for lymphocyte development in vivo. However, embryonic lethality of $\mathrm{Gcn}^{-/-}$and $\mathrm{Gcn} 5^{-/-P c a f^{--}}$mice (32) challenged the studies. To overcome the obstacle, we developed a complex mouse model, when Pcaf gene was germline-inactivated (32), while floxed Gcn5 gene (33) was conditionally-inactivated in B cell lineages by CRE recombinase expressed under Cd19 promoter (34). To sort out CRE-positive and CRE-negative cells, we used Rosa26-stop-YFP knockin which only expressed YFP following the CRE activation (35).

Here, we found that GCN5 and PCAF acetyltransferases are functionally redundant during early B cell maturation, while GCN5 is required for robust CSR.

\section{Materials and Methods}

\subsection{Mice}

Gcn5flf (33), Pcaft/- (32), Cd19Cre+ (34) (\# 006785; The Jackson Laboratory, Bar Harbor, ME, USA), Rosa26-stop-YFP+ (35)(\# 006148, The Jackson Laboratory, Bar Harbor, ME, USA), and $\mathrm{Aid}^{-/-}$(36) mice were previously described. Mice used for experiments were 
between 8-12 weeks of age. All experiments were performed in compliance with the Danish Working Environment Authority, the Danish Animal Experiment Inspectorate, the Department of Experimental Medicine (University of Copenhagen), and the Animal Resources Care Facility of Norwegian University of Science and Technology (NTNU, Trondheim, Norway).

\subsection{Flow cytometry}

Flow cytometry experiments were performed as we described earlier $(25,28,37-39)$. In particular, we used the fluorescent antibodies recognizing the proteins described below. B220 (PE-CF594, FITC, Alexa Fluor 700; all clone RA3-6B2, BD Bioscience, Franklin Lakes, NJ, USA). IgM (PerCP-eFluor 710, APC-eFluor 780, APC, FITC; all clone II/41, eBioscience, Santa Clara, CA, USA). IgG1 (PE, MOPC-21, Biolegend, SanDiego, CA, USA). IgG3 (PE, MG3-35, Biolegend, San Diego, CA, USA). CD3-APC (Biolegends, USA, \#100312). CD19 (Alexa Fluor 700, APC eFluor 780, both clone 1D3, eBioscience, Santa Clara, CA, USA). CD43 (APC and PE-Cy7, both clones S7, BD Bioscience, Franklin Lakes, NJ, USA).

\subsection{Class switch recombination}

The CSR was performed as we described earlier $(15,27,38-42)$.

\subsection{Western blot}

The western blot procedures were performed as we described earlier $(28,37,38,43)$. Briefly, the cells were lysed for 30 minutes on ice in Radioimmunoprecipitation assay (RIPA) buffer (Sigma Aldrich, St. Louis, MO, USA, \#R0278) supplemented with cOmplete $^{\mathrm{TM}}$ EDTA-free Protease Inhibitor Cocktail (Sigma Aldrich, \#11873580001). Proteins were analyzed by $4-12 \%$ Bis-Tris NuPAGE gels (Invitrogen, Carlsbad, CA, USA, \#NP0322), transferred to PDVF membranes (GE Healthcare, Boston, MA, USA, \#GE10600023), and probed with indicated antibodies. Rat anti-AID (1:500, Active Motif, Carlsbad, CA, USA, \#39886); mouse anti-GCN5, clone A-11 (1:500, Santa Cruz Biotechnology, Dallas, TX, USA, \#sc-365321); rabbit anti-PCAF, clone C14G9 (1:1000, Cell Signaling Technology, Leiden, The Netherlands, \#3378); rabbit anti-histone H3 (1:1000, Abcam, Cambridge, UK, \#ab1791); rabbit anti-histone H3 acetyl K9 (1:500, Abcam, Cambridge, UK, \#ab32129).

\subsection{Statistics}

We performed statistical analyses with one-way ANOVA using GraphPad Prism 8.0.1.244 (San Diego, CA, USA). In the tests, $p$ values less than 0.05 were defined as significant, i.e. ${ }^{*} \mathrm{p}<0.05 ;{ }^{* *} \mathrm{p}<0.01 ;{ }^{* * *} \mathrm{p}<0.001$; and ${ }^{* * * *} \mathrm{p}<0.0001$.

\section{Results}

\subsection{Generation of mice lacking GCN5 and PCAF in B cells}

Combined inactivation of Gcn5 and Pcaf genes in mice results in embryonic lethality (32). To overcome this challenge, we designed a complex genetic model when floxed Gcn5 gene is conditionally inactivated in B cell lineages by CRE enzyme under the Cd19 promoter $\left(C d 19^{C r e+}\right)(34)$. To sort out the cells with activated CRE, we used a model with knocked-in yellow fluorescent protein gene (YFP) into ROSA-26 locus. The YFP is inactive until CRE removes "STOP" signal (Rosa-26-YFP+) (35). Thus, we obtained Gcn5fff caf $^{\prime-}$

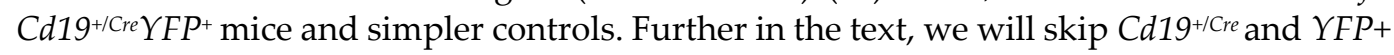
for simplicity in most of the cases, and will refer to the mice based on the status of Gcn5 and Pcaf genes, i.e. as Gcn5ffPcaf/, Gcn5ffl, Pcaf/- and WT. When the CRE is active and describing sorted B cells, we indicate $\mathrm{Gcn5}^{-/}$, a knockout status of the gene. Lack of GCN5 and PCAF, as well as H3K9 acetylation, was validated using western blot (e.g., Figure S1).

\subsection{Mice lacking GCN5 and PCAF in B cells possess small spleens}


We obtained mice with germline inactivation of $P$ caf gene and conditional inactivation of Gcn5 in B cells under the Cd19 promoter (Figure 1). We found that germline inactivation of Pcaf gene alone has no detectable effect on mouse development, in line with the previous observation (32). Conditional inactivation of Gcn5 gene in B cells had no visible effect on sizes of WT and Pcaf-deficient mice, which were 15 to $19 \mathrm{~g}$ on average $(\mathrm{p}>0.1433)$ (Figure 1A). However, inactivation of Gcn5 resulted in smaller spleens in mice (Gcn5/-, 54 $\mathrm{mg})$, when compared to WT (69 mg) and $\mathrm{Pcaf}^{\prime-}(72 \mathrm{mg})$ mice. Combined inactivation of Pcaf and Gcn5 in B cells resulted in even smaller spleens (Gcn5/-Pcaf/-, 29mg, p<0.0001). Spleens of mice without CRE activity with Gcn5 gene being floxed and functional (Gcn5f/fPcaf/-, $67 \mathrm{mg})$, were comparable in size to the ones of WT (Figure $1 \mathrm{~B}, \mathrm{C}$ ).
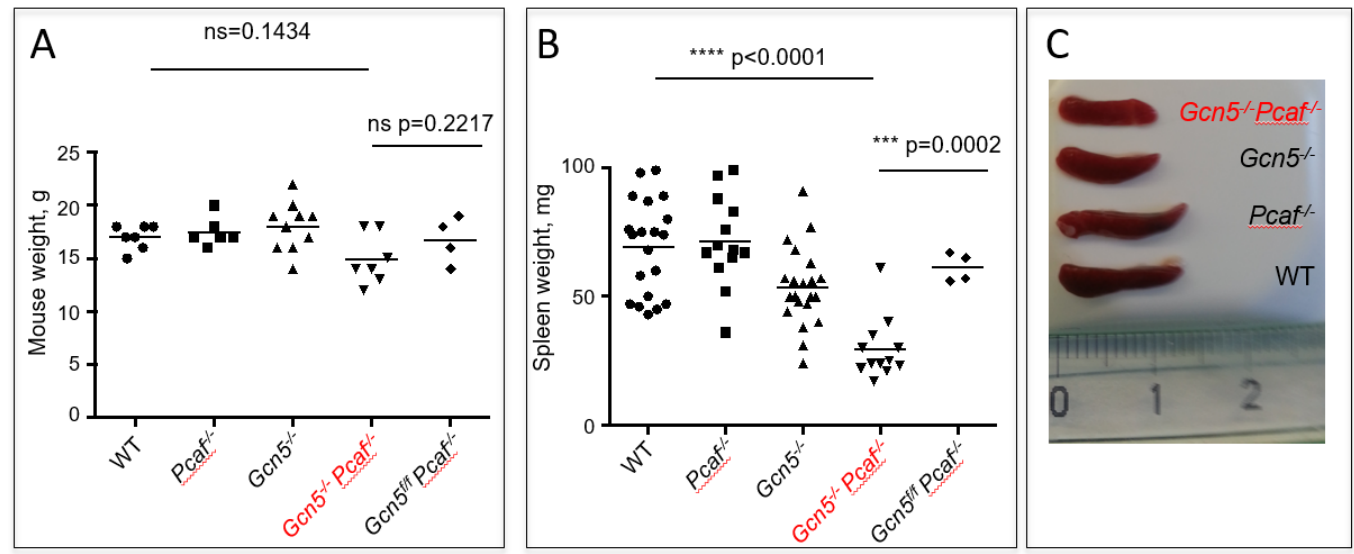

Figure 1. Generation of mice with germline inactivation of Pcaf and conditional inactivation of Gcn5 in B cell lineages. (A) Sizes of 8 weeks-old mice of indicated genotype are similar ( $>0.1433$ ). (B) Size of spleens from mice of indicated genotypes. WT vs Pcaf ${ }^{\prime-}$, n.s. $\mathrm{p}=0.9559$; WT vs Gcn $5^{-1}$,

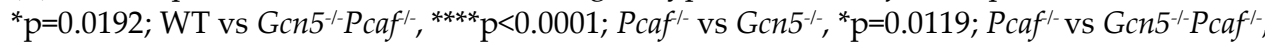
${ }_{* * * *} \mathrm{p}<0.0001 ; G c n 5^{-/-}$vs Gcn5 ${ }^{--P}$ caf ${ }^{\prime-},{ }^{* * *} \mathrm{p}=0.0008$. (C) Example of spleens of indicated genotypes. $\mathrm{Gcn}^{-/-}$indicates $\mathrm{Cd} 19^{\mathrm{Cre}}$-dependent inactivation of Gcn5 in B cell lineages.

\subsection{Mice lacking GCN5 and PCAF in B cells possess delayed B lymphocyte development}

To detect mature Gcn5-/-Pcaf/- B cells, we identified B220+IgM+ cells in the spleen using flow cytometry (Figure 2 A, B). Inactivation of Pcaf gene alone did not affect B cell proportions in the spleen (58\%) when compared to WT mice $(52 \%, \mathrm{p}=0.4777)$ (Figure $2 \mathrm{~A})$. Inactivation of Gcn5 alone resulted in an insignificant reduction of mature splenocytes when compared to WT (Gcn5\% have a significantly less B cell frequency in the spleen $(21 \%, \mathrm{p}<0.0001)$. Similarly, the numbers of Gcn5 ${ }^{--P}$ caf ${ }^{-}$B splenocytes was the lowest (3,4 million), while the number of Gcn5 $5^{-1}$ B cells (27 million) was also reduced when compared to $P$ caf ${ }^{\prime-}\left(54\right.$ million, $\left.{ }^{* *} \mathrm{p}=0.0065\right)$ and WT (44 million, ${ }^{*} \mathrm{p}=0.0214$ ) controls (Figure 2B). 

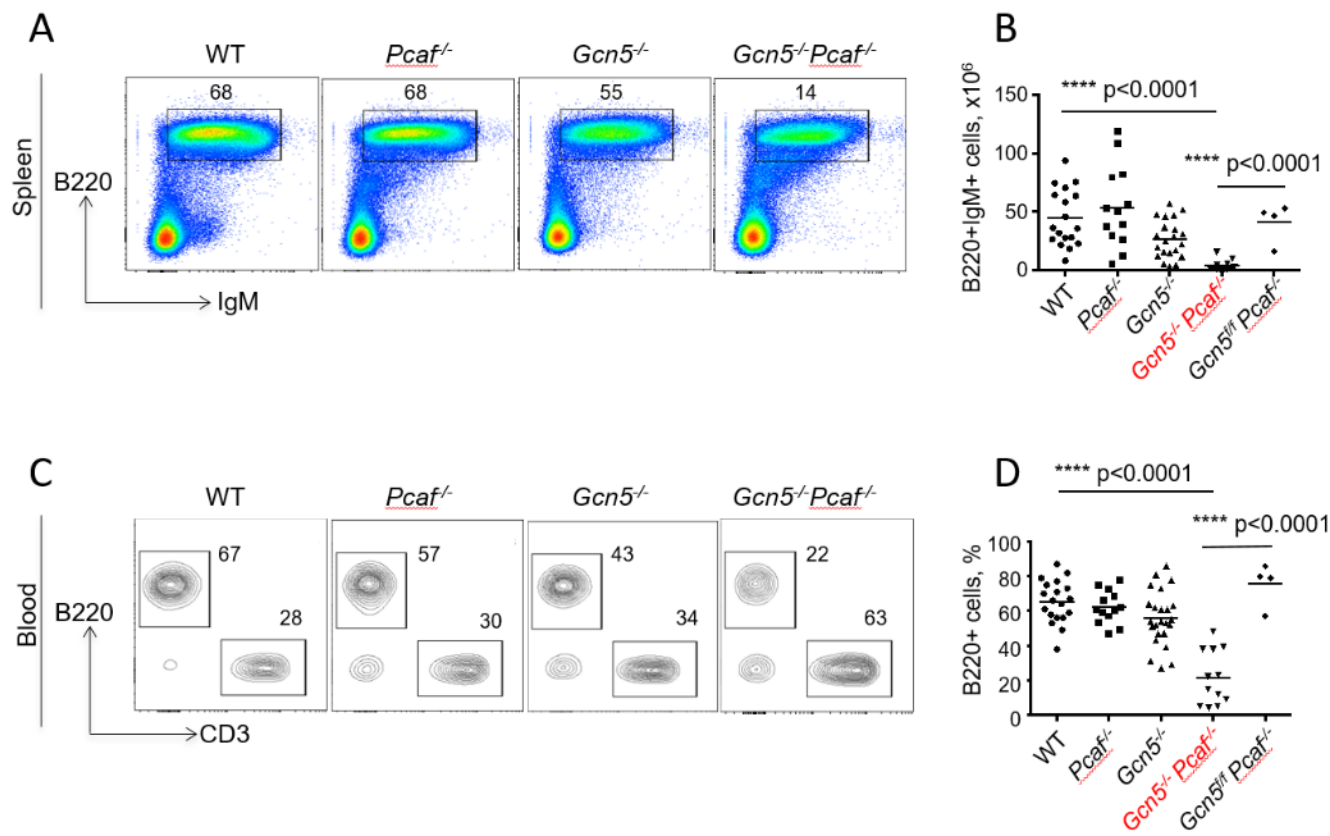

Figure 2. Reduced levels of mature B lymphocytes in spleens and blood of 8-12-weeks-old mice of indicated genotypes. (A) Proportions of B220+IgM+ mature B cells in spleen. WT vs Pcaf ${ }^{\prime}$, n.s.

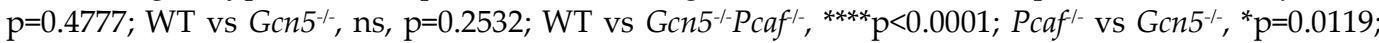

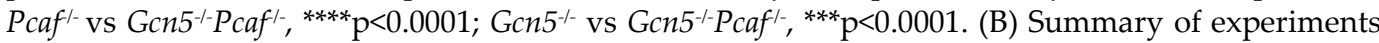
as shown in (A), number of cells. WT vs Pcaf/, n.s., $\mathrm{p}=0.9212$; WT vs Gcn5 ${ }^{-/}$, * $\mathrm{p}=0.0214$; WT vs Gcn5 ${ }^{-/}$

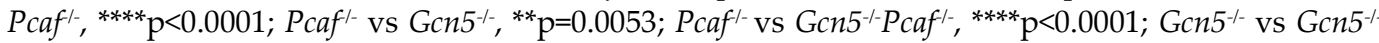
Pcaf'-, * $<<0.0269$. (C) Proportions of B220+ B cells and CD3+ T cells in blood. (D) Summary of several experiments from (C) reflecting proportions of B cells in blood. WT vs Pcafl-, n.s. $\mathrm{p}=0.9196$; WT vs Gcn5 ${ }^{-/}$, n.s., $\mathrm{p}=0.1204$; WT vs Gcn5 ${ }^{-/} P c a f^{\prime-}$, **** $\mathrm{p}<0.0001 ; P_{c a f^{\prime-}}$ vs Gcn5 $5^{-/}$, n.s., $\mathrm{p}=0.5398 ;$ Pcaf $^{\prime-}$ vs Gcn5

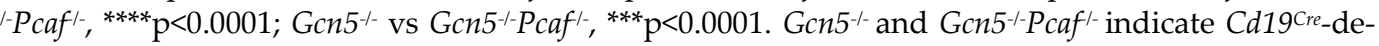
pendent inactivation of Gcn5 in B cell lineages.

\subsection{Inactivation of Gcn5 and Pcaf results in a reduced proportion of B cells in the blood}

To detect mature B cells in the blood, we used B220 markers (Figure 2C, D). Inactivation of Pcaf alone resulted in $62 \%$ of B cells after red blood cells were lysed, which was comparable to WT mice with $65 \%$ of B cells in the blood ( $\mathrm{p}=0.92)$. Inactivation of $G c n 5$ gene alone resulted in a modest reduction of $B$ cell proportion to $56 \%(p=0.12)$, while combined inactivation of Gcn 5 and Pcaf led to even lower B cell levels in blood (22\%, $\mathrm{p}<0.0001)$. Levels of B cells in blood of control mice without CRE recombinase expression, when Gcn5 gene was functional $\left(\mathrm{Gcn} 55^{f f} \mathrm{Pcaf}{ }^{\prime}\right)$ were comparable to WT mice (78\%). We conclude that GCN5 and PCAF are both required and functionally redundant for B cell development in mice.

One reason for low Gcn5--Pcaf`- B cell count in spleen and blood in mice could be cell death following normal development of $B$ cells in bone marrow and migration to periphery. Another option could be blocked or delayed maturation of $B$ cells in bone marrow during the earlier developmental stages. To test the latter possibility, we analyzed B cells in bone marrow of the mice (Figure 3).

\subsection{Inactivation of Gcn 5 and Pcaf results in accumulation of pro-B cells in bone marrow}

To characterize $B$ cell maturation in bone marrow, we followed the expression of B220 (B220+IgM-) and IgM (B220+IgM+) on the lymphocyte surface. Inactivation of Gcn5 or Pcaf resulted in an insignificant decline of B220+IgM+ population (26\%, 5-6 million) when compared to WT (32\%, 8 million, $\mathrm{p}=0.44)$ (Figure $3 \mathrm{~A}, \mathrm{~B})$. Combined inactivation of Gcn 5 and Pcaf resulted in an additional reduction of mature B cells in bone marrow (3 million, 16\%) (Figure $3 \mathrm{~A}, \mathrm{~B}$ ).

We further focused on B220+IgM- populations by determining the CD43+ (pro-B cells) and CD43- (pre-B cells). Proportion of early-stage pro-B cells increased from WT mice 
( $20 \%$ on average) and Pcaf/- mice ( $26 \%, \mathrm{p}=0.78)$ to $\mathrm{Gcn}^{-/-}\left(39 \%,{ }^{* *} \mathrm{p}=0.0022\right)$ to Gcn5 ${ }^{-/}$Pcaf ${ }^{\prime}$ $(55 \%, \mathrm{p}<0.0001)$ (Figure $3 \mathrm{C}, \mathrm{D})$. However, the number of pro-B cells in bone marrow estimated using our method of cell extraction was rather stable, with 0.8 million for WT $(n=13)$

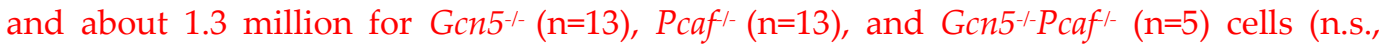
$\mathrm{p}>0.3830$ ). It suggested that the proportion of $G c n 5^{-}-\mathrm{Pcaf} /$ - pro-B cells was increased because the total number of mature B cells was reduced (Supplemental Figure S3 and Figure 3B).

We conclude that GCN5 and PCAF are required for the maturation of $\mathrm{B}$ cells from the pro-B cell stage to pre-B and later to mature B cells.
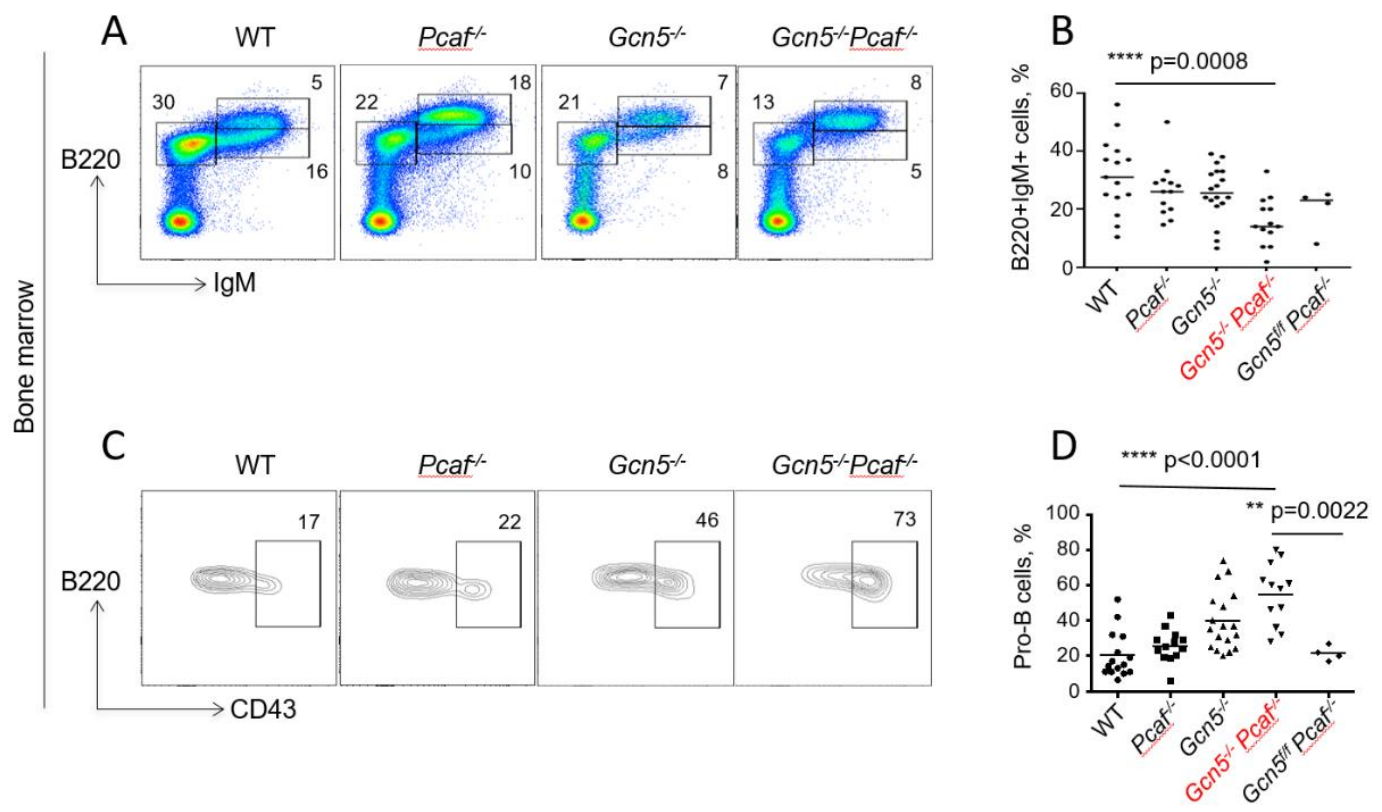

Figure 3. Delayed development of GCN5/PCAF-deficient B cells in bone marrow. Flow cytometry analyses of developing $B$ lymphocytes in bone marrow of 8-12-week-old mice of indicated genotypes. (A) Examples of B220+IgM- (pro-B and pre-B), B220+IgM+low (immature B) and B220+IgM+high (mature B) cell populations. (B) Summary of several experiments from (A) indicating B220+IgM+ cells. WT vs Pcaf ${ }^{\prime-}$, n.s. $\mathrm{p}=0.6445$; WT vs Gcn5 $5^{-/}$, n.s., $\mathrm{p}=0.4432$; WT vs Gcn5 ${ }^{-1-P}$ caf ${ }^{\prime-}$,

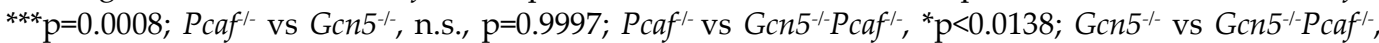
${ }^{*} \mathrm{p}<0.0211$ (C) CD43+ (pro-B cells) and CD43- (pre-B cells) gated from B220+IgM- populations in (A). (D) Summary of several experiments detecting B220+IgM-CD43+ (pro-B) cells. . WT vs Pcaf/-, n.s.

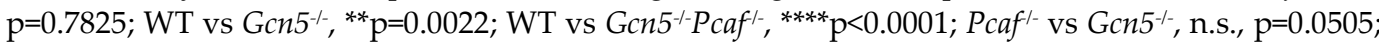

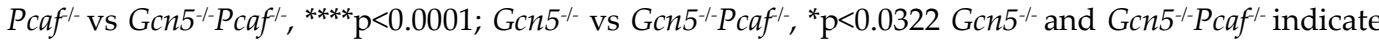
Cd19Cre-dependent inactivation of Gcn5 in B cell lineages.

\subsection{GCN5 is required for robust class switch recombination}

The CSR relies on the ATM-dependent DDR $(2,4,5,27)$. Because H3K9 acetylation works downstream of H2AX phosphorylation, and GCN5/PCAF might work downstream of ATM/ATR/DNA-PKcs, we tested if the CSR depends on GCN5 and PCAF (Figure 4). We purified B splenocytes from 8 to 12 weeks old mice and stimulated the CSR from IgM to IgG3 using established protocols $(40,42)$. We focused on matched pairs of Gcn5fff (the functional equivalent of WT cells) and $\mathrm{Gcn}^{-/}$, as well as Gcn5ffPcaf $\mathrm{f}^{\prime-}$ (the functional equiv-

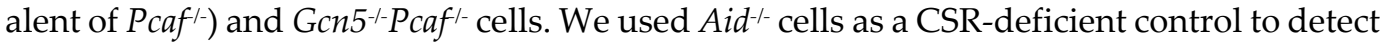
an experimental background (Figure 4). Inactivation of Pcaf alone had no effect on CSR levels (WT vs Gcn5ffPcaf/, p p 0.96). Contrary, the inactivation of Gcn5 resulted in a reduction of CSR from about $14 \%$ in WT and Gcn5ff cells to $6 \%$ in Gcn5 ${ }^{-/}$cells, ${ }^{*} \mathrm{p}<0.0008$ (Figure 4). Combined deletion of Pcaf and Gcn5 resulted in a similar reduction from $12 \%$ in Gcn ${ }^{f f}$ Pcaf $^{\prime}$ - cells to $6 \%$ in Gcn5 ${ }^{-}-$Pcaf ${ }^{\prime}$ - cells, ${ }^{* *} \mathrm{p}=0.0080$. We conclude that GCN5 is required for robust CSR to IgG3 because additional inactivation of Pcaf did not affect CSR levels when compared Gcn5 ${ }^{-/}$and Gcn5 ${ }^{-/}$Pcaf/- B cells, p>0.9999 (Figure 4). 


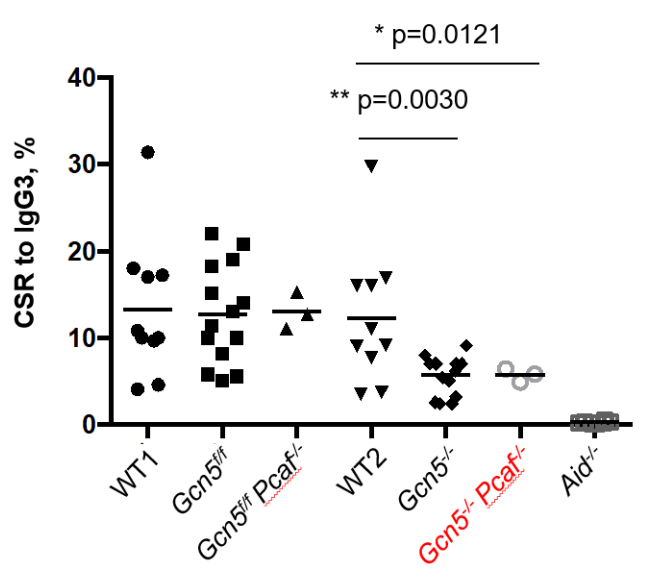

Figure 4. Class switch recombination of stimulated mature B cells from IgM to IgG3. Gcn5 ${ }^{-/-}$and Gcn5 $5^{-/} \mathrm{Pcaf} /$ - indicate $C d 19^{C r e}$-dependent inactivation of Gcn5 in B cell lineages. The levels of CSR for WT, Gcn5 flf, Gcn $5^{f / f} \mathrm{Pcaf} /$ are not significantly different (n.s.); the levels for $\mathrm{Gcn} 5^{-1-}$ and $\mathrm{Gcn}^{-1-P c a f}{ }^{-}$are lower than the former three groups ( $p=0.0030$ and $p=0.0121$, correspondently, when compared to WT2); Gcn5 ${ }^{-/}$vs Gcn5 $5^{-1-P c a f}{ }^{--}$levels are similar ( $\mathrm{p}=0.9997)$, and the Aid $^{-/}$has only background levels, WT2 vs Aid $^{-/}$is $(\mathrm{p}>0.0001)$.

\section{Discussion}

Both GCN5 and PCAF are involved in chromatin modification and DDR response, which made them relevant candidates to facilitate lymphocyte development $(13,14)$. One challenge was the lack of a relevant in vivo model because GCN5 and PCAF have certain redundant functions in acetylating $\mathrm{H} 3 \mathrm{~K} 9$, and because the germline inactivation of Gcn5 results in early embryonic lethality in mice (32). Here, we generated and analyzed a complex mouse model which allowed studying of double-deficient Gcn5 $5^{-/}$Pcaf - $^{-}$B cells development in vivo and ex vivo. We used a germline knockout of Pcaf (32), a conditional knockout of Gcn5ff, a knockin of CRE recombinase expressed under the B cell-specific Cd19 promoter (34), and a knockin of YFP to track the activity of CRE recombinase (35).

For such a complex mouse model (Gcn5fffPcaf/Cd19+/cre Rosa-26-YFP+), multiple controls were used. In one line of the controls, the mice lacking PCAF, having floxed Gcn5 gene but expressing no CRE recombinase were considered (Gcn fff Pcafl-Rosa-26-YFP+, Figure S2). The GCN5-deficient and GCN5/PCAF double-deficient B cells possessed developmental delay with lower levels of mature B cells in spleen and blood, and accumulation of progenitor B cells in bone marrow (Figures 1-4). Contrary, the control mice without CRE expression demonstrated WT levels of $B$ cell development in all the groups, i.e. WT levels of B220+IgM+ mature B cells in the spleen (Figure S2 A and B) and blood (Figure 2S $C$ and D). In addition, these mice possessed high levels of B220+IgM+ cells (Figure S2 E, $\mathrm{F})$, as well as stable and low levels of pro-B cells (B220+IgM-CD43+) in bone marrow (Figure $\mathrm{S} 2, \mathrm{G}$, and $\mathrm{H}$ ).

The mice lacking PCAF and with conditional knockout of Gcn5 in B cells were alive and resembled WT littermates (Figure 1A). One clear feature the Gcn $5^{f f f} \mathrm{Pcaf} f^{\prime} \mathrm{C} d \mathrm{C}^{+/ \text {cre }} \mathrm{Rosa}$ 26-YFP+ mice had was a small spleen (Figure $1 \mathrm{~B}$ and $\mathrm{C}$ ), which was also the case in mice lacking only Gcn 5 in B cells. The small spleen could indicate a defect in B cell development, and we indeed found low numbers of mature $B$ cells in the spleen, blood, and bone marrow. One could propose that mature B cells lacking GCN5 or both GCN5 and PCAF possess low proliferation speed or tend to trigger apoptosis. Alternatively, GCN5 and PCAF might be required for the $\mathrm{V}(\mathrm{D}) \mathrm{J}$ recombination. This option could be tested by, for example, using vAbl pre-B cell lines as we and others did before, e.g. $(4,15,18,23,27-31)$. Another intriguing question is whether the physical presence or enzymatic activity of GCN5 and PCAF are required for the observed phenotypes, i.e. abrogated B cell maturation and reduced levels of CSR. To investigate this question one could use specific inhibitors of GCN5 and PCAF enzymes, or enzyme-dead mutations introduced to the Gcn5 and Pcaf genes.

Inactivation of Gcn5 in murine B cells also resulted in reduced lymphomagenesis in mice overexpressing MYC oncoprotein (44). Our findings further highlight this observation, suggesting that GCN5, and potentially also PCAF, enzymes are attractive targets for cancer therapy (44). 
The CSR levels were reduced in B cells lacking GCN5 (Figure 4). The challenge in this set of experiments was that the mice of Gcn5ffPcafl-Cd19+/cre Rosa-26-YFP+ genotype were rather rare and possessed a very low number of suitable B splenocytes (Figures 1 and 2). Although our data on IgG3 is sufficient, one could extend the study in the future by generating knockout cell lines lacking GCN5 and PCAF and suitable for CSR. One possible model system is $\mathrm{CH} 12 \mathrm{~F} 3$ cells capable to support CSR to IgA (45), which were used in the past for this kind of experiment $(24,37,42)$. The CSR itself is a complex multistage process. Generating relevant cell lines will also provide tools to determine specific stages of CSR affected in GCN5-deficient mice, i.e. germline transcription, AID recruitment, generation of DSBs, or DNA repair.

\section{Conclusions}

Acetyltransferases GCN5 and PCAF possess redundant functions in B cell maturation. GCN5 is required for robust class switch recombination ex vivo.

Author Contributions: Conceptualization, methodology, validation, formal analysis, investigation, resources, data curation - J.A.D., D.S., and V.O.; writing - original draft preparation, V.O.; writing review and editing, V.O. and J.A.D.; supervision, J.A.D.; project administration, J.A.D. and V.O.; funding acquisition, J.A.D. and V.O. All authors have read and agreed to the published version of the manuscript.

Funding: This research was mainly supported by the Novo Nordisk Foundation Grant NNF14CC0001 to J.A.D. In addition, V.O. was supported by The Research Council of Norway (\#249774, \#270491 and \#291217); the Norwegian Cancer Society (\#182355); The Health Authority of Central Norway (\#13477 and \#38811); and The Outstanding Academic Fellow Program at NTNU 2017-2021. The final part of the work was supported by the Stiftelsen Kristian Gerhard Jebsen (grant SKGJ-MED-019).

Acknowledgments. Gcn5-flox and Pcaf-null mice were kindly provided by the Sharon Dent laboratory.

Institutional Review Board Statement: All experiments were performed in compliance with the Danish Working Environment Authority, the Danish Animal Experiment Inspectorate, the Department of Experimental Medicine (University of Copenhagen), and the Animal Resources Care Facility of Norwegian University of Science and Technology (NTNU, Trondheim, Norway), FOTS \#11931 (2017) and FOTS \#13405 (2018).

Conflicts of Interest: The authors declare no conflict of interest. The funders had no role in the design of the study; in the collection, analyses, or interpretation of data; in the writing of the manuscript, or in the decision to publish the results.

\section{References}

1. C. Lescale, L. Deriano, The RAG recombinase: Beyond breaking. Mech Ageing Dev 165, 3-9 (2017).

2. F. W. Alt, Y. Zhang, F. L. Meng, C. Guo, B. Schwer, Mechanisms of programmed DNA lesions and genomic instability in the immune system. Cell 152, 417-429 (2013).

3. X. S. Wang, B. J. Lee, S. Zha, The recent advances in non-homologous end-joining through the lens of lymphocyte development. DNA Repair (Amst) 94, 102874 (2020).

4. S. Castaneda-Zegarra et al., Genetic interaction between the non-homologous end-joining factors during $B$ and $T$ lymphocyte development: In vivo mouse models. Scand J Immunol 92, e12936 (2020).

5. V. Kumar, F. W. Alt, V. Oksenych, Functional overlaps between XLF and the ATM-dependent DNA double strand break response. DNA Repair (Amst) 16, 11-22 (2014).

6. R. L. Frock, C. Sadeghi, J. Meng, J. L. Wang, DNA End Joining: G0-ing to the Core. Biomolecules 11 (2021).

7. D. L. Bordin, L. Lirussi, H. Nilsen, Cellular response to endogenous DNA damage: DNA base modifications in gene expression regulation. DNA Repair (Amst) 99, 103051 (2021). 
8. T. Saha, D. Sundaravinayagam, M. Di Virgilio, Charting a DNA Repair Roadmap for Immunoglobulin Class Switch Recombination. Trends Biochem Sci 46, 184-199 (2021).

9. X. Zhang et al., Fundamental roles of chromatin loop extrusion in antibody class switching. Nature 575, 385-389 (2019).

10. Y. Zhang et al., The fundamental role of chromatin loop extrusion in physiological V(D)J recombination. Nature 573, 600-604 (2019).

11. D. Menolfi, S. Zha, DNA-PKcs kinase activity orchestrates both end-processing and end-ligation. Trends Cell Biol 10.1016/j.tcb.2021.12.002 (2021).

12. K. Ragunathan, N. L. E. Upfold, V. Oksenych, Interaction between Fibroblasts and Immune Cells Following DNA Damage Induced by Ionizing Radiation. Int J Mol Sci 21 (2020).

13. H. S. Lee, J. H. Park, S. J. Kim, S. J. Kwon, J. Kwon, A cooperative activation loop among SWI/SNF, gamma-H2AX and H3 acetylation for DNA double-strand break repair. EMBO J 29, 1434-1445 (2010).

14. Q. Jin et al., Distinct roles of GCN5/PCAF-mediated H3K9ac and CBP/p300-mediated H3K18/27ac in nuclear receptor transactivation. EMBO J 30, 249-262 (2011).

15. V. Oksenych et al., Functional redundancy between the XLF and DNA-PKcs DNA repair factors in V(D)J recombination and nonhomologous DNA end joining. Proc Natl Acad Sci U S A 110, 2234-2239 (2013).

16. M. Xing, M. Bjoras, J. A. Daniel, F. W. Alt, V. Oksenych, Synthetic lethality between murine DNA repair factors XLF and DNA-PKcs is rescued by inactivation of Ku70. DNA Repair (Amst) 57, 133-138 (2017).

17. M. Xing, V. Oksenych, Genetic interaction between DNA repair factors PAXX, XLF, XRCC4 and DNA-PKcs in human cells. FEBS Open Bio 9, 1315-1326 (2019).

18. V. Abramowski et al., PAXX and Xlf interplay revealed by impaired CNS development and immunodeficiency of double KO mice. Cell Death Differ 25, 444-452 (2018).

19. G. Balmus et al., Synthetic lethality between PAXX and XLF in mammalian development. Genes Dev 30, $2152-2157$ (2016).

20. S. Castaneda-Zegarra, M. Xing, R. Gago-Fuentes, S. Saeterstad, V. Oksenych, Synthetic lethality between DNA repair factors Xlf and Paxx is rescued by inactivation of Trp53. DNA Repair (Amst) 73, 164-169 (2019).

21. C. Lescale et al., Specific Roles of XRCC4 Paralogs PAXX and XLF during V(D)J Recombination. Cell Rep 16, 2967-2979 (2016).

22. X. Liu, Z. Shao, W. Jiang, B. J. Lee, S. Zha, PAXX promotes KU accumulation at DNA breaks and is essential for end-joining in XLF-deficient mice. Nat Commun 8, 13816 (2017).

23. P. J. Hung et al., Deficiency of XLF and PAXX prevents DNA double-strand break repair by non-homologous end joining in lymphocytes. Cell Cycle 16, 286-295 (2017).

24. V. Kumar, F. W. Alt, R. L. Frock, PAXX and XLF DNA repair factors are functionally redundant in joining DNA breaks in a G1-arrested progenitor B-cell line. Proc Natl Acad Sci U S A 113, 10619-10624 (2016).

25. S. Castaneda-Zegarra et al., Leaky severe combined immunodeficiency in mice lacking non-homologous end joining factors XLF and MRI. Aging (Albany NY) 12, 23578-23597 (2020).

26. P. J. Hung et al., MRI Is a DNA Damage Response Adaptor during Classical Non-homologous End Joining. Mol Cell 71, 332342 e338 (2018).

27. S. Zha et al., ATM damage response and XLF repair factor are functionally redundant in joining DNA breaks. Nature 469, 250-254 (2011).

28. C. Beck, S. Castaneda-Zegarra, C. Huse, M. Xing, V. Oksenych, Mediator of DNA Damage Checkpoint Protein 1 Facilitates V(D)J Recombination in Cells Lacking DNA Repair Factor XLF. Biomolecules 10 (2019).

29. B. R. Chen et al., The RNF8 and RNF168 Ubiquitin Ligases Regulate Pro- and Anti-Resection Activities at Broken DNA Ends During Non-Homologous End Joining. DNA Repair (Amst) 108, 103217 (2021).

30. X. Liu et al., Overlapping functions between XLF repair protein and 53BP1 DNA damage response factor in end joining and lymphocyte development. Proc Natl Acad Sci U S A 109, 3903-3908 (2012). 
31. V. Oksenych et al., Functional redundancy between repair factor XLF and damage response mediator 53BP1 in V(D)J recombination and DNA repair. Proc Natl Acad Sci U S A 109, 2455-2460 (2012).

32. W. Xu et al., Loss of Gcn512 leads to increased apoptosis and mesodermal defects during mouse development. Nat Genet 26, 229-232 (2000).

33. W. Lin et al., Developmental potential of Gcn5(-/-) embryonic stem cells in vivo and in vitro. Dev Dyn 236, 1547-1557 (2007).

34. R. C. Rickert, J. Roes, K. Rajewsky, B lymphocyte-specific, Cre-mediated mutagenesis in mice. Nucleic Acids Res 25, 13171318 (1997).

35. S. Srinivas et al., Cre reporter strains produced by targeted insertion of EYFP and ECFP into the ROSA26 locus. BMC Dev Biol 1, 4 (2001).

36. M. Muramatsu et al., Class switch recombination and hypermutation require activation-induced cytidine deaminase (AID), a potential RNA editing enzyme. Cell 102, 553-563 (2000).

37. A. Dewan et al., Robust DNA repair in PAXX-deficient mammalian cells. FEBS Open Bio 8, 442-448 (2018).

38. R. Gago-Fuentes et al., Normal development of mice lacking PAXX, the paralogue of XRCC4 and XLF. FEBS Open Bio 8, 426434 (2018).

39. S. Castaneda-Zegarra et al., Generation of a Mouse Model Lacking the Non-Homologous End-Joining Factor Mri/Cyren. Biomolecules 9 (2019).

40. L. M. Starnes et al., A PTIP-PA1 subcomplex promotes transcription for IgH class switching independently from the associated MLL3/MLL4 methyltransferase complex. Genes Dev 30, 149-163 (2016).

41. D. Su et al., PTIP chromatin regulator controls development and activation of B cell subsets to license humoral immunity in mice. Proc Natl Acad Sci U S A 114, E9328-E9337 (2017).

42. C. Boboila et al., Robust chromosomal DNA repair via alternative end-joining in the absence of X-ray repair crosscomplementing protein 1 (XRCC1). Proc Natl Acad Sci U S A 109, 2473-2478 (2012).

43. R. Gago-Fuentes, V. Oksenych, Non-Homologous End Joining Factors XLF, PAXX and DNA-PKcs Maintain the Neural Stem and Progenitor Cell Population. Biomolecules 11 (2020).

44. A. T. Farria et al., Transcriptional Activation of MYC-Induced Genes by GCN5 Promotes B-cell Lymphomagenesis. Cancer Res 80, 5543-5553 (2020).

45. M. Nakamura et al., High frequency class switching of an IgM+ B lymphoma clone CH12F3 to IgA+ cells. Int Immunol 8, 193201 (1996). 